\title{
LARGE SCALE CAVITIES SURROUNDING MICROQUASARS INFERRED FROM EVOLUTION OF THEIR RELATIVISTIC JETS
}

\author{
J. F. Hao ${ }^{1}$ and S. N. Zhang ${ }^{1,2,3}$ \\ jingfang.hao@hotmail.com,zhangsn@mail.tsinghua.edu.cn \\ ${ }^{1}$ Department of Physics and Tsinghua Center for Astrophysics, Tsinghua University, Beijing \\ 100084, China \\ ${ }^{2}$ Key Laboratory of Particle Astrophysics, Institute of High Energy Physics, Chinese Academy of \\ Sciences, Beijing 100049, China \\ ${ }^{3}$ Physics Department, University of Alabama in Huntsville, Huntsville, AL 35899, USA
}

\begin{abstract}
The black hole X-ray transient XTE J1550-564 has undergone a strong outburst in 1998 and two relativistic X-ray jets have been detected years later with the Chandra $\mathrm{X}$-ray observatory; the eastern jet was found previously to have decelerated after its first detection. Here we report a full analysis of the evolution of the western jet; significant deceleration is also detected in the western side. Our analysis indicates that there is a cavity outside the central source and the jets first traveled with constant velocity and then were slowed down by the interactions between the jets and the interstellar medium (ISM). The best fitted radius of the cavity is $\sim 0.31 \mathrm{pc}$ on the eastern side and $\sim 0.44$ pc on the western side, and the densities also show asymmetry, of $\sim 0.034 \mathrm{~cm}^{-3}$ on the east to $\sim 0.12 \mathrm{~cm}^{-3}$ on the west. The best fitted magnetic fields on both sides are $\sim 0.5$ mG. Similar analysis is also applied to another microquasar system, H 1743-322, and a large scale low density region is also found. Based on these results and the comparison with other microquasar systems, we suggest a generic scenario for microquasar jets, classifying the observed jets into three main categories, with different jet morphologies (and sizes) corresponding to different scales of vacuous environments surrounding them. We also suggest that either continuous jets or accretion disk winds, or both may be responsible for creating these cavities. Therefore X-ray jets from microquasars provide us with a promising method of probing the environment of accreting black holes.
\end{abstract}

Subject headings: jets and outflows - radiation mechanisms: nonthermal- accretion disks - black hole physics -stars: individual (XTE J1550-564, H 1743-322, GRS 1915+105, GX 339-4) -X-rays: stars 


\section{INTRODUCTION}

Microquasars are well known miniatures of quasars, with a central black hole (BH), an accretion disk and two relativistic jets very similar to those found in the centers of active galaxies, only on much smaller scales (Mirabel \& Rodríguez 1999). The typical timescales in these systems are also $10^{5}-10^{7}$ times shorter than those in quasars, thus evolutions of microquasar jets can be studied in details. Therefore, microquasar systems have been considered ideal laboratories for understanding accretion process in black hole systems and might provide us a good alternative to study AGN phenomena instead of observing them directly (Massi \& Kaufman Bernadó 2008).

Since discovered in 1992, radio jets have been observed in a series of BH binary systems and several of them showed apparent superluminal features. In the two well known microquasars, GRS 1915+105 (Mirabel \& Rodríguez 1999) and GRO J1655-40 (Tingay et al. 1995; Hjellming \& Rupen 1995), relativistic jets with actual velocities greater than $0.9 c$ were observed. In some other systems, small-size "compact jets", e.g. Cyg X-1 (Stirling et al. 2001), and large scale diffuse emission, e.g. SS433 (Dubner et al. 1998), were also detected.

Among these microquasars, XTE J1550-564 is especially interesting since it was the first Galactic accretion systems that a fast moving X-ray jet was detected. The large scale and long existing time of the jets have also made this source unique and valuable in jet studies. XTE J1550-564 was discovered with RXTE in 1998 during its strong X-ray outburst on September 7 (Smith 1998). It is believed to be an X-ray binary system at a distance of $\sim 5.3 \mathrm{kpc}$, containing a black hole of $10.5 \pm 1.0$ solar masses and a low mass companion star (Orosz et al. 2002). Soon after the discovery of the source, a jet ejection with an apparent velocity greater than $2 c$ was reported by VLBI team (Hannikainen et al. 2001). In the period between 1998 and 2002, the source also exhibited strong $\mathrm{X}$-ray activities but no similar radio and X-ray flares were detected again during these activities (Tomsick et al. 2003).

With the Chandra satellite, Corbel et al. (2002) found two large scale X-ray jets lying to the east and west of the central source, which were also in good alignment with the central source. The eastern jet has been detected first in 2000 at a projected distance of $\sim 21$ arcsec and a position angle $\mathrm{E}$ of $\mathrm{N}$ of $93.8^{\circ} \pm 0.9^{\circ}$ from the central black hole. Its apparent proper motion velocity has dropped from an average of $\sim 32.9$ mas/day between 1998 and 2000 to $\sim 21.2$ mas/day during its 2000 observations; its X-ray flux also decayed quite rapidly. Two years later, it could only be seen marginally in the X-ray image, while a western counterpart became visible at $\sim 22$ arcsec on the other side. The corresponding radio maps are consistent with the X-ray observations (Corbel et al. 2002).

From the year 2002 to 2003, there were altogether five observations of this source in the Chandra archive. The eastern jet has disappeared from the X-ray images in late 2002, while the western jet could be seen clearly in all the five observations, although the flux decayed quickly as well. Among these observations, only the first two have been reported and discussed previously (Kaaret et al. 2003; Wang et al. 2003). We therefore use these additional data points to better 
constrain the kinematic and spectral analysis for the eastern jet, and to extend the analysis to the western jet.

In this paper, we first describe the observational data obtained by Chandra X-ray satellite in section 2 and 3. Then we propose a model for the system and discuss the kinematic and emission process in section 4 . Finally in section 5 and 6, we apply this model to another source H 1743-322 and make some further discussions based on these analysis.

\section{OBSERVATIONS of XTE J1550-564}

There are altogether eight 2-dimensional imaging observations of XTE J1550-564 in the Chandra archive during June 2000 and October 2003 (henceforth observations 1 8). These observations were all made by the Advanced CCD Imaging Spectrometer (ACIS). There are also grating observations providing 1-dimensional imaging in the archive; but since our interests are in the jet kinematics analysis, these are not our focus at this stage.

We have down-loaded all data of these eight observations from the archive and examined the level 2 event files produced by Standard Data Processing procedures to make our analysis. Exposure time for these observations ranges from a few kiloseconds (observations 1 3) to a few tens of kiloseconds (observations 4 8) (Table 1). Background light curves are extracted for each of the observations and only in observation 5 a high background flare is detected. Except for this period, almost all valid exposure time intervals of these observations are included in our work.

In the section 2 and 3, we limit most of our analysis to the kinematic and spectral properties of the western jet. The central accreting source has already attracted much attention in recent years and there were already plenty of studies in details in literature (Corbel et al. 2001; Jain et al. 2001; Kubota \& Makishima 2004; Yuan et al. 2007). The eastern jet has also been studied fully in 2003 when it was discovered (Tomsick et al. 2003; Kaaret et al. 2003). As a result, we do not discuss much of our results for observations 1 3 here; only comparisons with the previous works will be noted for consistency. A complete study of the kinematic and light curve evolution of the western jet is our main goal in these two sections.

We list the basic information of observations $1 \sim 8$ in Table 1, including the observation ID, date, the positions of the central point source and the eastern and western jets respectively (in J2000 coordinate). The positions are obtained by the Chandra Interactive Analysis of Observations (CIAO) routine wavdetect (Freeman et al. 2002), a commonly used program in determining X-ray sources in Chandra images. From this table, we could see clearly that an X-ray emission source is detected to the east of the central source in the first four observations and another source is detected to the west in the last five observations. Calculations also show that these two sources, when presented in a single combined image, are in good alignment with the central compact object with a position angle $\mathrm{E}$ of $\mathrm{N}$ of $-85.9^{\circ} \pm 0.3^{\circ}$. In observations 5 and 6 , no X-ray source is detected by wavdetect at the position of the eastern jet. However, from the Gaussian smoothed images 
(Fig. 2), a weak source could be recognized in observation 6. We thus select the strongest emission region of this source manually with a small circle of the radius of 0.7 arcsec and use the position of its center as one data point in our further analysis.

In Fig.1, the raw, unsmoothed images of the last five observations are shown. An extended and moving source can be seen easily in these images. Its elongation points towards the central source, and has thus indicated clearly the relation between them. In most cases the flux from this western source is comparable to the central source; however in observation 5 , the observed surface brightness from the western source is even higher than the central source (also see section 3).

With the data in Table 1, we can calculate the angular separations between the jets and the central source as well as the average proper motions. In this paper, we have adopted the assumption that both the eastern and western jets are related to the same outburst in September 1998. Except for the outburst occurred in 1998, there were some other outbursts detected afterwards, including a major outburst in 2000 and some mini-outbursts in 2001 to 2003 (Tomsick et al. 2003 and references therein). However, the long term RXTE-ASM light curve shows that the X-ray flux of the 1998 outburst is four times higher than that of the 2000 outburst and more than twenty times higher than that of all the rest (Sturner \& Shrader 2005). The complex behaviors found in the 1998 outburst, such as irregular light curves and multiple state transitions, were not detected in other outbursts. The two X-ray jets are also found to be aligned with the 1998 radio jets (Corbel et al. 2002), which is also consistent with the assumption of a common origin of the two jets, but by itself does not provide definite evidence for the assumption unless the source is precessing strongly. Based on the above arguments, we conclude that it is reasonable to assume that both the eastern and western jets originated from the same 1998 outburst.

Our calculation results are listed in Table 2. A distance of approximately 23 arcsec is obtained for the western jet with a slowly increasing value; errors are estimated following the method described by Tomsick et al. (2003). We calculate the source centroid for the central source and the X-ray jet respectively and for all the five observations, the changes of the newly calculated position with the previous results are less than $0.5^{\prime \prime}$. Therefore, an upper limit of $0.5^{\prime \prime}$ is set for the error of the jet distance (except the eastern jet in observation 6, where a larger error of 1 arcsec was set because of the manual selection method we applied). We divide the net angular separation by the time interval between the neighboring observations to estimate the average proper motion for the jets, and from the results, an approximate estimate of deceleration could be seen for both jets. The absolute astrometry of Chandra is calibrated by fixing the X-ray position of the central source as its radio position reported by Corbel et al (2001).

\section{ENERGY SPECTRUM and FLUX}

We extract the X-ray spectrum in 0.3-8 keV energy band for each observation. To be consistent with previous analysis of the eastern jet, we follow some of those previous procedures. Because of 
Table 1. XTE J1550-564 Chandra Observations

\begin{tabular}{|c|c|c|c|c|c|c|c|}
\hline \multirow[b]{2}{*}{$\begin{array}{l}\text { Obs } \\
\text { Num }\end{array}$} & \multirow[b]{2}{*}{$\begin{array}{l}\text { Obs } \\
\text { ID }\end{array}$} & \multirow[b]{2}{*}{$\begin{array}{l}\text { Obs } \\
\text { date }\end{array}$} & \multirow[b]{2}{*}{$\begin{array}{c}\text { Exposure } \\
(\mathrm{s})\end{array}$} & \multicolumn{2}{|c|}{ Eastern Jet } & \multicolumn{2}{|c|}{ Western Jet } \\
\hline & & & & $\begin{array}{c}\text { RA } \\
(15: 51:)\end{array}$ & $\begin{array}{c}\text { Dec } \\
(-56: 28:)\end{array}$ & $\begin{array}{c}\text { RA } \\
(15: 50:)\end{array}$ & $\begin{array}{c}\text { Dec } \\
(-56: 28:)\end{array}$ \\
\hline 1 & 679 & 2000 Jun 9 & 3816 & $01 .^{\mathrm{a}}$ & 36.7 & & \\
\hline 2 & 1845 & 2000 Aug 21 & 5160 & $01.4 \pm 2 \times 10^{-2}$ & $36.7 \pm 1 \times 10^{-2}$ & & \\
\hline 3 & 1846 & 2000 Sep 11 & 4630 & $01.5 \pm 2 \times 10^{-2}$ & $36.6 \pm 1 \times 10^{-2}$ & & \\
\hline 4 & 3448 & 2002 Mar 11 & 26118 & $02.1 \pm 1 \times 10^{-2}$ & $37.4 \pm 1 \times 10^{-2}$ & $56.0 \pm 4 \times 10^{-3}$ & $33.5 \pm 2 \times 10^{-3}$ \\
\hline 5 & 3672 & 2002 Jun 19 & 18025 & & & $55.9 \pm 4 \times 10^{-3}$ & $33.7 \pm 2 \times 10^{-3}$ \\
\hline 6 & 3807 & 2002 Sep 24 & 24442 & $02.17^{\mathrm{b}}$ & 37.6 & $55.86 \pm 6 \times 10^{-3}$ & $33.9 \pm 3 \times 10^{-3}$ \\
\hline 7 & 4368 & 2003 Jan 28 & 23680 & & & $55.82 \pm 6 \times 10^{-3}$ & $33.6 \pm 4 \times 10^{-3}$ \\
\hline 8 & 5190 & 2003 Oct 23 & 47831 & & & $55.74 \pm 6 \times 10^{-3}$ & $33.6 \pm 3 \times 10^{-3}$ \\
\hline
\end{tabular}

${ }^{\text {a }}$ Positions of the eastern and the western jets are corrected after calibration of the central source to the position of the radio observation as $\mathrm{RA}=15: 50: 58.71$ and $\mathrm{DEC}=-56: 28: 35.7$ (Corbel et al. 2001) and the positions are subject to the 0.3 arcsec positional uncertainty found by Corbel et al. (2001) on their radio position. The quoted errors are the statistical errors provided by wavdetect. However, wavdetect did not provide error information for observation 1.

${ }^{\mathrm{b}}$ The position of the eastern jet in observation 6 is determined by the center of the strongest emission region, as marked by a small circle on fig 2 .

Table 2. Angular Separations and Proper Motions of the Eastern and Western Jets

\begin{tabular}{|c|c|c|c|c|c|c|}
\hline \multirow[b]{2}{*}{ Obs Num } & \multirow[b]{2}{*}{ Obs ID } & Time after & \multicolumn{2}{|c|}{ Separations to the $\mathrm{BH}(\operatorname{arcsec})^{\mathrm{a}}$} & \multicolumn{2}{|c|}{ Average Proper Motion (mas/d) } \\
\hline & & X-ray burst (days) & Eastern Jet & Western jet & Eastern Jet & Western jet \\
\hline 1 & 679 & 628 & $21.5 \pm 0.5$ & & $33.9 \pm 0.8$ & \\
\hline 2 & 1845 & 700 & $22.8 \pm 0.5$ & & $21.1 \pm 0.7$ & \\
\hline 3 & 1846 & 720 & $23.4 \pm 0.5$ & & $31.5 \pm 0.7$ & \\
\hline 4 & 3448 & 1265 & $28.6 \pm 0.5$ & $22.6 \pm 0.5$ & $9.4 \pm 0.4$ & $17.9 \pm 0.4$ \\
\hline 5 & 3672 & 1365 & & $23.2 \pm 0.5$ & & $6.3 \pm 0.4$ \\
\hline 6 & 3807 & 1462 & $29.2 \pm 1.4$ & $23.4 \pm 0.5$ & $3.3 \pm 0.3$ & $1.6 \pm 0.3$ \\
\hline 7 & 4368 & 1588 & & $23.7 \pm 0.5$ & & $2.2 \pm 0.3$ \\
\hline 8 & 5190 & 1856 & & $24.5 \pm 0.5$ & & $3.2 \pm 0.3$ \\
\hline
\end{tabular}

The error bar of the angular separation of the eastern jet in observation 6 is estimated as 2 times the radius of the circle.

${ }^{\mathrm{a}}$ Errors are estimated by following Tomsick et al. (2003).

${ }^{b}$ Values obtained by dividing the net angular separation and the error by the time interval between the neighboring observations. 

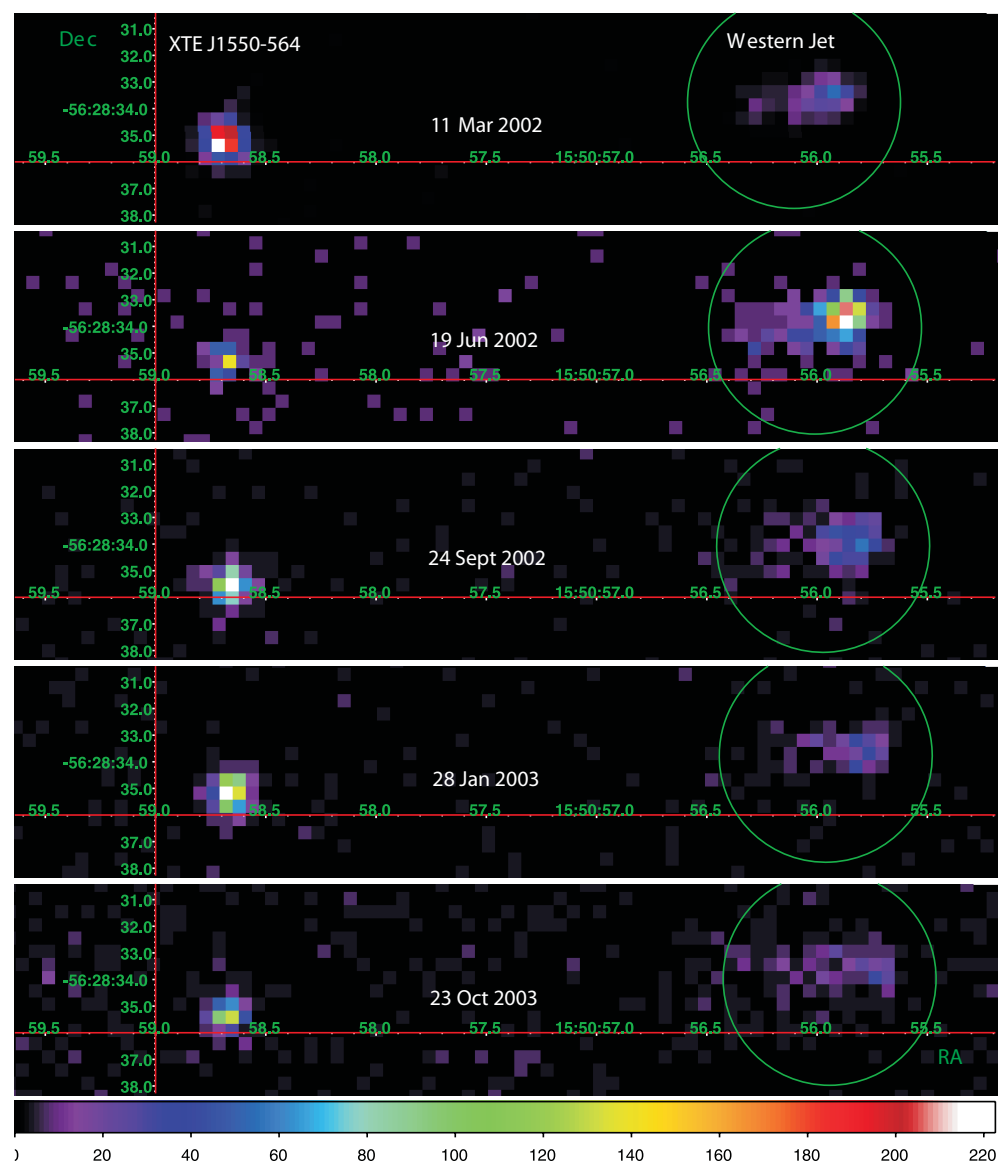

Fig. 1.- The Chandra 0.3-8 keV raw image showing XTE J1550-564 and the western jet. The images are in linear scale and no count saturation has been set. Each image is normalized to its own color scale. The color bar below the images indicates the relative strength. The numbers under the color bar indicate the number of photons in each pixel for the first image (obs 4, ID=3448), and the peak count values in the other four images (obs 5-8) are 27, 60, 58, 36, respectively. The pixel size is $0 " .492 \times 0$ ". 492 . The green circle in each observation shows the spectral extraction region for each jet, which is of 8 arcsec in diameter and includes almost all of the jet counts (albeit that there is no guarantee that all jet photons are included, due to the wing of the point spread function of the Chandra mirror assembly.). The images are aligned by RA (x-axis). 


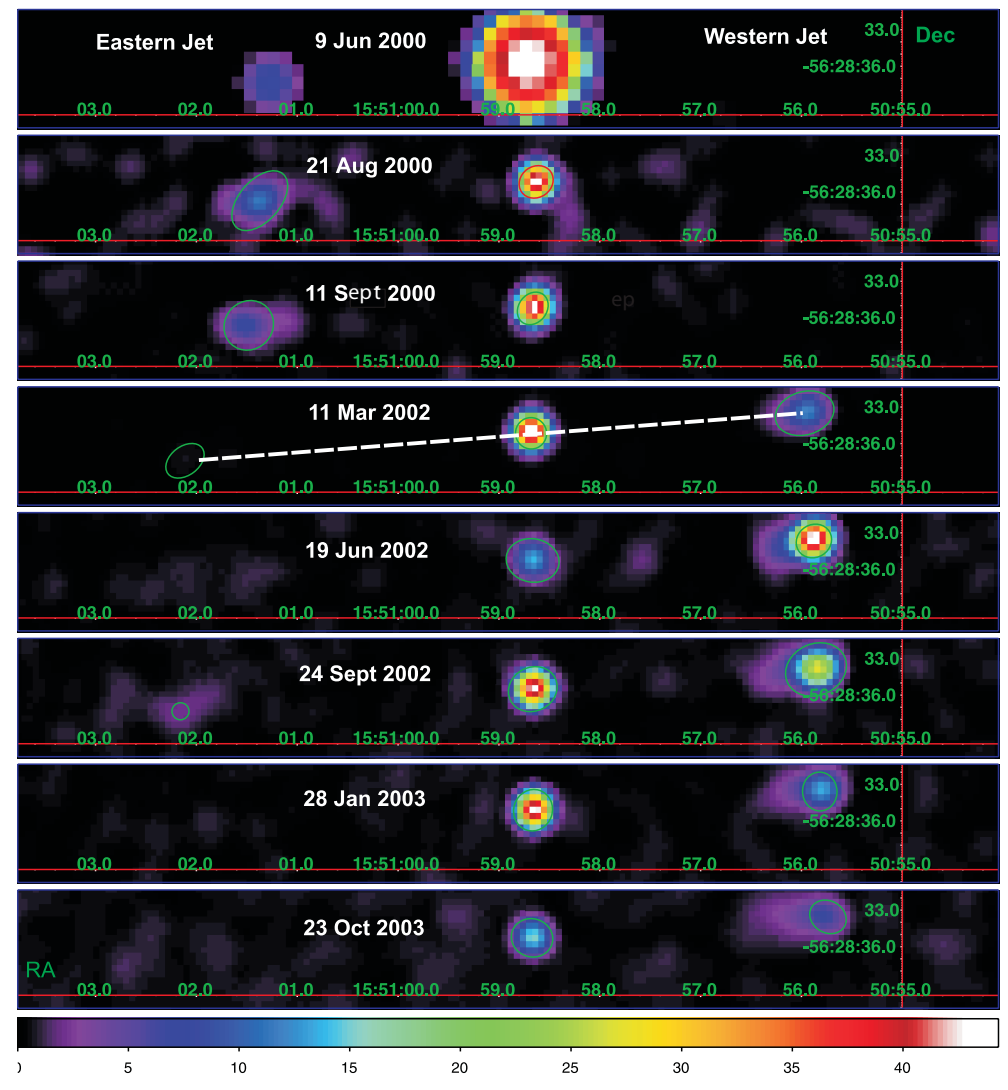

Fig. 2. - The smoothed Chandra X-ray images of the eight observations of XTE J1550-564 and the two jets together. Except for the first observation, in which the central $\mathrm{BH}$ is too bright so a log scale is set to reveal the eastern jet, all the images are in linear scale and no count saturation has been set. The appearance and disappearance of the eastern and western jets can be seen clearly in the image. Each image is normalized to its own color scale. The color bar below the images indicates the relative strength. The numbers under the color bar indicate the number of photons in each pixel for the fourth image (obs $4, \mathrm{ID}=3448$ ) (to be consistent with fig. 1), and the peak count values in the other seven images (obs 1-3, 5-8) are 18.8, 3.8, 6.3, 10.3, 13.4, 15.2, 9.0, respectively. The green elliptical regions are source emission regions detected by wavdetect. Observation 4 shows the good alignment of the two jets and the central source. The images are aligned by RA (x-axis). 
the much longer exposure time, the total counts collected from each of the last five observations are much more than those from the first three. We use a circular source region with a radius of $4^{\prime \prime}$, an annular background region with an inner radius of $5^{\prime \prime}$ and an outer radius of $15^{\prime \prime}$, for each observation. The photon number inside each selected region is about a few hundreds, possible for spectral analysis, compared to only $\sim 20$ counts in the jet region in observations 1,2 and 3 . We perform background subtraction but did not make independent background spectra.

Instrument response matrices (rmf) and weighted auxiliary response files (warf) are created using CIAO programs mkacisrmf and mkwarf and used in the fitting in Xspec. We re-bin the spectra with 10 counts per bin and fit them in Xspec. Several spectral models are tested, but the statistical quality is not high enough to distinguish between them completely. Taking observation 4 for example (for the reason that the photon number of this observation is the largest), synchrotron model (powerlaw) and self Compton scattering (compsl) seem to work equally well with the corresponding reduced $\chi^{2}$ of 0.924 and 0.925 . Their combination (powerlaw + compsl) does not improve the fitting $\left(\chi^{2} \sim 0.981\right)$, but creates a large uncertainty for each parameter (the best fitting photon index is $2 \pm 28$ in the combined model).

In table 3, the results of spectra fitting to the western jet with an absorbed power-law model are shown (spectra shown in Fig. 3). The absorption column density is fixed to the Galactic value in the direction of XTE J1550-564 obtained by the radio observations $\left(N_{H}=9 \times 10^{21} \mathrm{~cm}^{-2}\right)$ (Dickey \& Lockman 1990). The first two observations (obs. 4 \& 5) have been analyzed by Kaaret et al. (2003), and our results are quite consistent with their work. The mean photon index of the five observations is around 1.8, also consistent with the previous work on the eastern jet (Tomsick et al. 2003). A slightly different photon index is obtained for observation 6 and 8 ; however, the value of 1.8 is still within the $1-\sigma$ error range. The calculated absorbed energy flux in $0.3-8 \mathrm{keV}$ band is comparable to the value of the eastern jet. The observed flux decayed from $\sim 1.9 \times 10^{-13} \mathrm{erg}$ $\mathrm{cm}^{-2} \mathrm{~s}^{-1}$ in March 2002 to only one sixth of this value in October 2003 (see section 4.2). For observation 6 , we also try to fit the spectrum of the eastern jet. However, the data points are just too few to yield a satisfactory result. With only 17 counts between 0.3 to $10 \mathrm{keV}$ inside a 8 -arcsec-diameter circle, we obtained a reduced $\chi^{2}$ of only 0.13 when using the chi-squared statistic in fitting. When changing to the Cash statistic, a more suitable method for low counts cases, the resulting photon index is 1.11 and the estimated flux is $1.6 \times 10^{-14} \mathrm{erg} \mathrm{cm}^{-2} \mathrm{~s}^{-1}$, even higher than the value in observation 4 . We consider this result not very convincing, or the re-heating process is more complicated than a simple shock. Complete understanding of this problem is beyond the scope of the current work. As a result, we drop this spectral data point in the light curve fitting.

\section{JET MODEL}

We now describe the model of Wang et al. (2003), and apply it to the data, demonstrating how it needs to be revised to fit the full set of observations of the eastern and western jets. The kinematic and light curve models are constructed according to the external shock model in the 


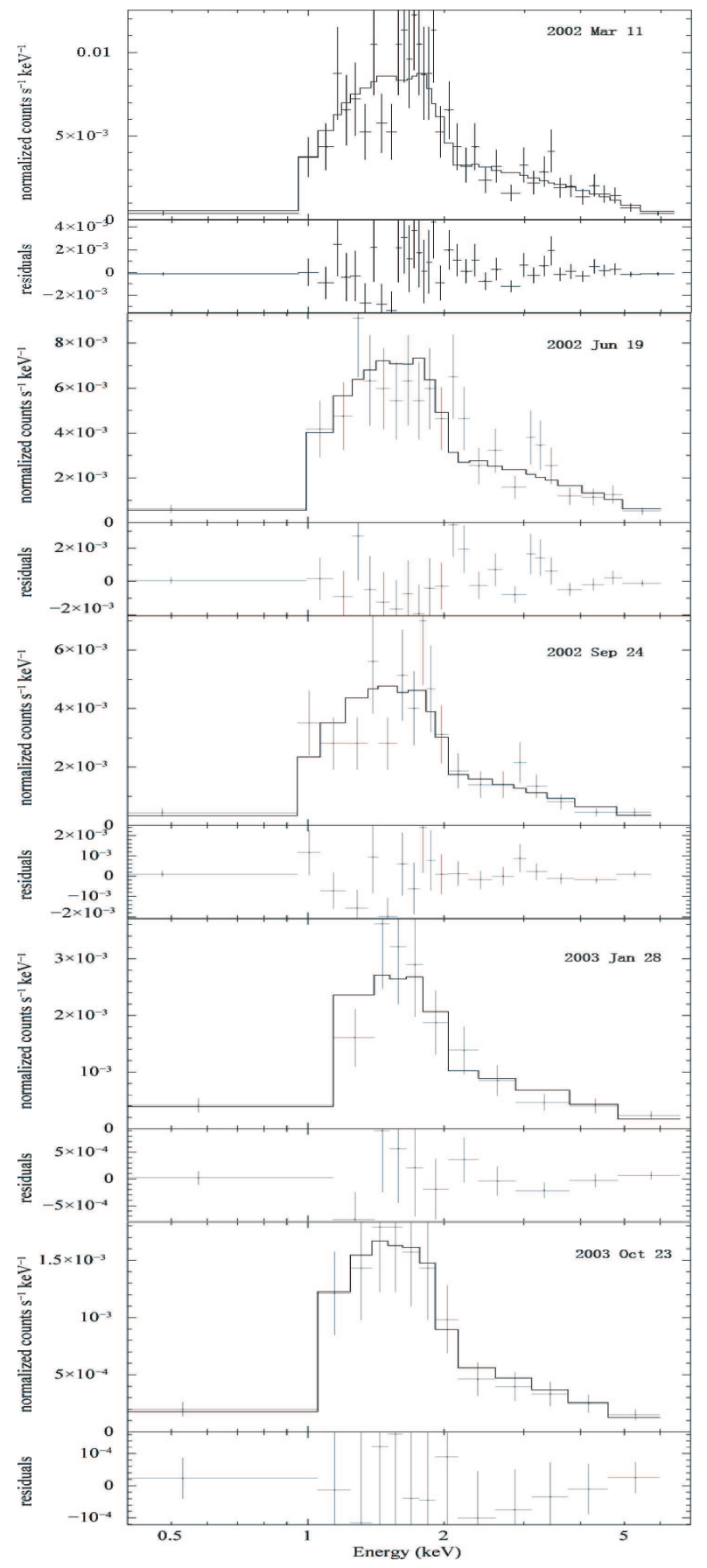

Fig. 3.- X-ray spectra of emission from the western jet during March 2002 and October 2003. The five panels are data from observations $4 \sim 8$ respectively. An absorbed power-law model with the neutral hydrogen column density fixed to the Galactic value, $9 \times 10^{21} \mathrm{~cm}^{-2}$, is used in the fitting. Values of all fitted parameters are listed in Table 3. 
standard gamma-ray burst (GRB) afterglow theory. In this model, the kinematic evolution and light curve of the jets can be interpreted by the interactions between the jet materials and the interstellar medium (ISM). The relativistic ejecta losses its energy to the ISM and is therefore slowed down to non-relativistic phase. In Wang et al. (2003), they used the reverse shock emission model to fit the light curve of the eastern jet successfully. We therefore apply this model to the analysis of the western jet as well.

\subsection{Kinematic Model}

As we know, in the external shock model for afterglows of GRBs, the kinematic and light curve evolution could be understood as the interaction between the outburst ejecta and the surrounding ISM (Rees \& Mészáros 1992). Since microquasar systems are also Galactic sources embedded inside the ISM, we may infer that the jets of microquasars should also encounter such interactions and thus be decelerated during their expansion. The large scale and long existing time of XTE J1550-564 jets have provided us a good opportunity to test this scenario.

Because of the good alignment of the two jets and the central accreting object, we adopt the model of a collimated conical beam with a half opening angle $\theta_{j}$ expanding into the ambient medium with the number density $n$. The initial kinematic energy and Lorentz factor of the outflow material are $E_{0}$ and $\Gamma_{0}$, respectively. Shocks should arise as the outflow moves on and heat the ISM, and its kinematic energy will turn into the internal energy of the medium gradually. Neglect the radiation loss, the energy conservation equation writes (Huang, Dai, \& Lu 1999):

$$
(\Gamma-1) M_{0} c^{2}+\sigma\left(\Gamma_{\mathrm{sh}}^{2}-1\right) m_{\mathrm{SW}} c^{2}=E_{0} .
$$

The first term on the left of the equation represents the kinematic energy of the ejecta, where $\Gamma$ is the Lorentz factor and $M_{0}$ is the mass of the original ejecta. The second term represents the internal energy of the swept-up ISM, where $\Gamma_{\text {sh }}$ and $m_{\mathrm{SW}}$ are the corresponding Lorentz Factor and mass of the shocked ISM respectively, and

$$
m_{\mathrm{SW}}=V_{\mathrm{g}} m_{\mathrm{p}} n\left(\theta_{j}^{2} / 4\right),
$$

with $V_{\mathrm{g}}$ as the shocked gas volume. Coefficient $\sigma$ differs from $6 / 17$ to 0.73 for ultra-relativistic and non-relativistic jets (Blandford \& McKee 1976). Wang et al. (2003) took $\sigma \sim 0.7$ and we adopt their approximation in our work. Equation (1) and the relativistic kinematic equations

$$
\left(\frac{d R}{d t}\right)_{\mathrm{a}}=\frac{\beta(\Gamma) c}{1-\beta(\Gamma) \cos \theta} ;\left(\frac{d R}{d t}\right)_{\mathrm{r}}=\frac{\beta(\Gamma) c}{1+\beta(\Gamma) \cos \theta}
$$

can be solved and give the relation between the projected angular separation $\mu$ and time $t$. In equations (2), the subscript ' $a$ ' and ' $r$ ' represent the approaching and receding jets in a pair of

relativistic jets respectively. $R$ is the distance between the jet and the source, which can be 
transformed into the angular separation by $\mu=R \sin \theta / 5.3 \mathrm{kpc}$, and $\theta$ is the jet inclination angle to the line of sight. We can get the $\mu-t$ curve numerically with the above equations. To be consistent with the work done to the eastern jet, we test first the same initial conditions that $\Gamma_{0}=3, E_{0}=3.6 \times 10^{44} \mathrm{erg}, \theta=50^{\circ}$ and $\theta_{j}=1 .^{\circ} 5$ as those found by Wang et al. (2003) and would like to find how these parameters affect the fitting of the jet motions.

In the case of the eastern jet, the number density of the ISM was assumed by Wang et al. (2003) as a constant in the whole region outside the central source, thus the interaction was supposed to have taken place all the way along the jet's path. This assumption does not work well in the case of its western counterpart, because the western jet has traveled by 2002 as far as the eastern jet had traveled by 2000 , but was decelerated much more significantly. This can be seen in Fig. 4 . The western jet moved comparatively little after its appearance and acted like "hitting into a wall". This requires much stronger interactions and thus a much denser environment. We tried to fit the western jet itself but were not very successful. If we increase the number density, the gas will simply block the jet and the jet will never travel to such a far distance (a $10^{44}$ erg jet can only move to $\sim 7-8$ arcsec in $1 \mathrm{~cm}^{-3}$ ISM). On the other hand, if the initial energy is too large $\left(\sim 10^{50}\right.$ erg), the jet could penetrate ISM and travel far but would be very difficult to slow down so quickly (for such high energy, even a density of $100 \mathrm{~cm}^{-3}$ can not bend the curve down). Changing the initial Lorentz factor has similar results with changing initial energy and the opening angle of the jet affects the results quite little. This tendencies are plotted in Fig.4 (a), where the fitting to the eastern jet shows the model of Wang et al. (2003) and the corresponding solid-line fitting to the western jet uses the same model and same parameters. The dashed line and dot dashed line there represent the results of changing the number density or the initial kinetic energy to ten times of the eastern results. All three curves do not match the data points well.

As a result, we consider keeping the initial Lorentz factor and energy, while changing the geometric assumptions to describe the jet motions. For the inclination angle, we first examined the paper of Orosz et al. (2002), where they find a value of $72.6 \pm 4.8^{\circ}$ from the optical observations. We took this value, but varying it within their 1-sigma error bars, and find $\theta=68^{\circ}$ gives out the best fit. To simplify the problem, we just fix the inclination angle to this value in our work.

After setting the inclination angle, we test a model that the ISM density varies as the distance changes, i.e., the density is lower in the center and higher in the outside region. For simplicity, we test the case that the jet traveled first through a "cavity" with a constant velocity and then through a dense region and was decelerated there. For this modification, we introduce another parameter $r$, the outer radius of the cavity. The ISM number density is set to be a constant $n$ outside this region and zero inside. The results are shown in panel (b) and (c) in Fig.4. We first apply this model to the eastern and the western jet separately. The results are quite satisfactory but not well constrained. Because of only 10 data points in constraining 4 free parameters $\left(r_{\mathrm{e}}, r_{\mathrm{w}}, n_{\mathrm{e}}, n_{\mathrm{w}}\right)$, there are more than one group of parameters that seem work well. Fitting the two jets simultaneously with a fixed cavity radius helps a bit. It constrains the fitting tighter and $r=16$ arcsec seems an acceptable result for the both sides (Fig.4 (b)). However, this is not a sound assumption. The 
inferred ISM number density corresponding to this value is $0.015 \mathrm{~cm}^{-3}$ in the east and $0.06 \mathrm{~cm}^{-3}$ in the west respectively, indicating a clear asymmetry in the ISM density on the two side. Thus it is not reasonable to assume a perfect symmetric geometry. We still need other analysis to help determining consistent parameters.

\subsection{Light Curve Model}

In the standard GRB scenario, the afterglow emission is produced by the synchrotron radiation or inverse Compton emission of the accelerated electrons in the shock front of the jets. The forward shock emission is from the heated electrons in the swept-up ambient medium, whereas the reverse shock emission is from the electrons of the jet itself when a shock moves back through the ejecta. The same processes should also happen in the case of microquasars, with only the Lorentz factor much lower. In Wang et al. (2003), they tested these two possibilities and proved that the forward shock emission would decay too slowly to fit for the observed decay index $-3.7 \pm 0.7$ of the eastern jet, because of the continuously heating effect. However, since only operating once, the reverse shock emission could decay rather fast as the electrons cooled adiabatically and this model indeed performed quite well in describing the data. In the case of the western jet, the energy flux decayed even more rapidly (decay index $\sim-5.1 \pm 0.1$, see Fig. 5 ) and as a result, we take the reverse shock mechanism in our work.

Assuming the distribution of the electrons obeys a power-law form, $n\left(\gamma_{\mathrm{e}}\right) d \gamma_{\mathrm{e}}=K \gamma_{\mathrm{e}}^{-p} d \gamma_{\mathrm{e}}$, for $\gamma_{\mathrm{m}}<\gamma_{e}<\gamma_{\mathrm{M}}$, the volume emissivity at frequency $\nu^{\prime}$ in the comoving frame is given by (Rybicki \& Lightman 1979)

$$
j_{\nu^{\prime}}=\frac{\sqrt{3} q^{3}}{2 m_{\mathrm{e}} c^{2}}\left(\frac{4 \pi m_{\mathrm{e}} c \nu^{\prime}}{3 q}\right)^{\frac{(1-p)}{2}} B_{\perp}^{\frac{(p+1)}{2}} K F_{1}\left(\nu, \nu_{\mathrm{m}}^{\prime}, \nu_{\mathrm{M}}^{\prime}\right)
$$

where

$$
F_{1}\left(\nu, \nu_{m}^{\prime}, \nu_{M}^{\prime}\right)=\int_{\nu^{\prime} / \nu_{M}^{\prime}}^{\nu^{\prime} / \nu_{m}^{\prime}} F(x) x^{(p-3) / 2} d x
$$

with $F(x)=x \int_{x}^{+\infty} K_{5 / 3}(t) d t$ and $K_{5 / 3}(t)$ is the Bessel function. The physical quantities in these equations include $q$ and $m_{\mathrm{e}}$, the charge and mass of the electron, $B_{\perp}$, the magnetic field strength perpendicular to the electron velocity, and $\nu_{m}^{\prime}$ and $\nu_{M}^{\prime}$, the characteristic frequencies for electrons with $\gamma_{m}$ and $\gamma_{M}$.

Assuming the reverse shock heats the ejecta at time $t_{0}$ at the radius $R_{0}$ (with the assumptions of no synchrotron cooling, conservation of the total number of electrons and the magnetic field being frozen into the plasma), the physical quantities in the adiabatically expanding ejecta with radius $R$ evolve as (van der Laan 1966)

$$
\begin{gathered}
\gamma_{m}=\gamma_{m}\left(t_{0}\right) \frac{R_{0}}{R}, \gamma_{M}=\gamma_{M}\left(t_{0}\right) \frac{R_{0}}{R}, \\
K=K\left(t_{0}\right)\left(\frac{R}{R_{0}}\right)^{-(2+p)}, B_{\perp}=B_{\perp}\left(t_{0}\right)\left(\frac{R}{R_{0}}\right)^{-2},
\end{gathered}
$$



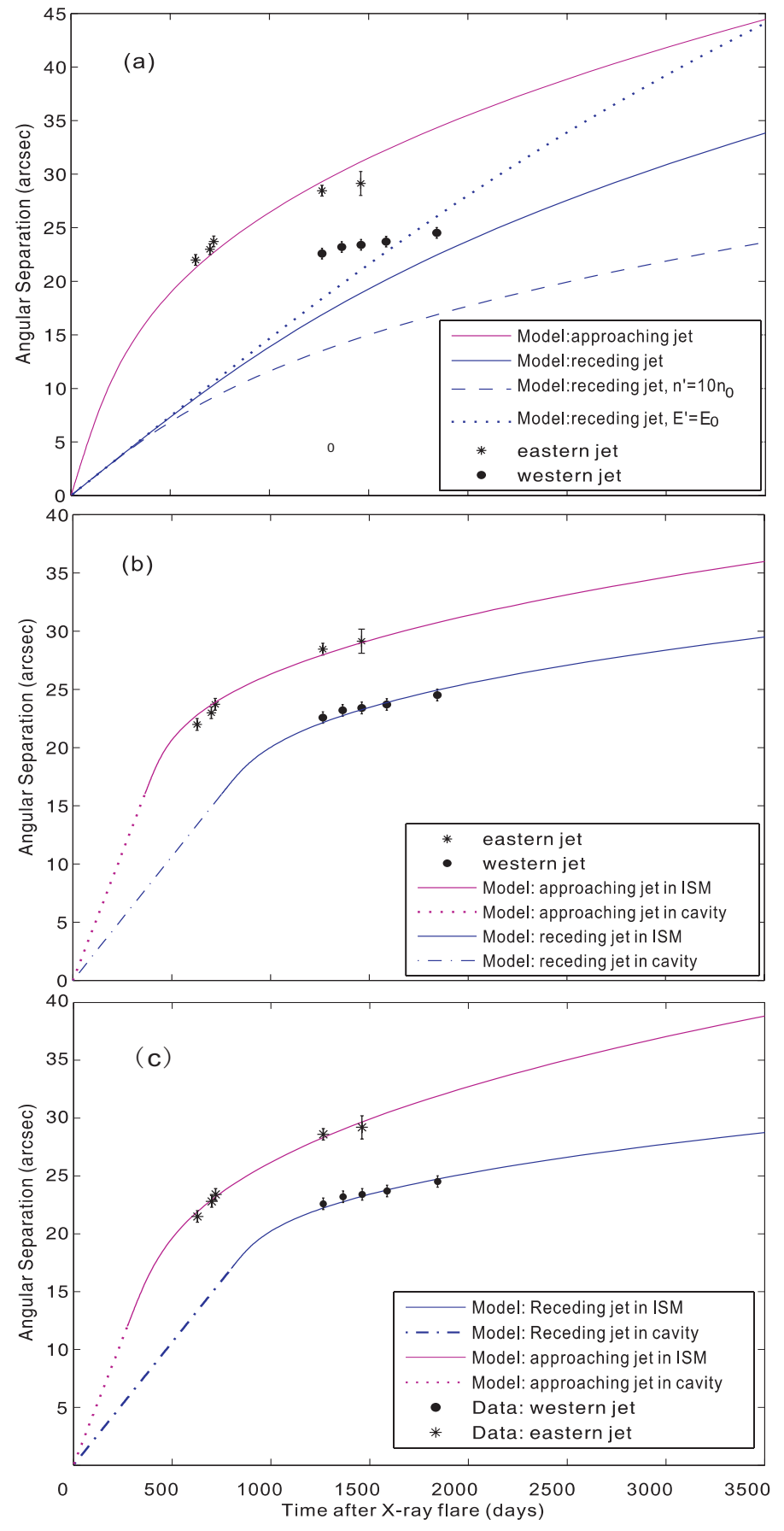

Fig. 4.- Model fittings to the proper motion of the X-ray jets of XTE J1550-564. In all three panels, the pink lines are the model predictions of the approaching jet and the blue lines are models predictions of the receding jet. Panel (a): $\theta=50^{\circ}, n_{0}=1.5 \times 10^{-4} \mathrm{~cm}^{-3}, E_{0}=3.6 \times 10^{44} \mathrm{erg}$ and $r_{\mathrm{e}}=r_{\mathrm{w}}=0$. Fitting to the approaching jet is the model from Wang et al. (2003). The model for the western jet is based on the same parameters. The dashed and dotted lines are the results if the number density or the initial kinematic energy is changed to ten times of the eastern results. Panel (b): model with a symmetric cavity introduced. Parameters: $\theta=68^{\circ}, r_{\mathrm{e}}=r_{\mathrm{w}}=16$ arcsec, $n_{\mathrm{e}}=0.015$ $\mathrm{cm}^{-3}, n_{\mathrm{w}}=0.06 \mathrm{~cm}^{-3}$. Panel (c): model with an asymmetric cavity introduced. Parameters: $\theta=68^{\circ}$, $r_{\mathrm{e}}=12 \operatorname{arcsec}, r_{\mathrm{w}}=17 \operatorname{arcsec}, n_{\mathrm{e}}=0.0034 \mathrm{~cm}^{-3}, n_{\mathrm{w}}=0.12 \mathrm{~cm}^{-3}$. 
where the initial values of these quantities are free parameters to be fitted in the calculation.

With these assumptions, we can then calculate the predicted flux evolution of the jets. The comoving frequency $\nu^{\prime}$ relates to our observer frequency $\nu$ by $\nu=D \nu^{\prime}$, where $D$ is the Doppler factor and we have $D_{\mathrm{a}}=1 / \Gamma(1-\beta \cos \theta)$ and $D_{\mathrm{r}}=1 / \Gamma(1+\beta \cos \theta)$ for the approaching and receding jets respectively. Considering the geometry of the emission region, the observed X-ray flux in $0.3-8 \mathrm{keV}$ band could be estimated by

$$
F(0.3-8 \mathrm{keV})=\int_{\nu_{1}}^{\nu_{2}}\left[\frac{\theta_{\mathrm{j}}^{2}}{4}\left(\frac{\mathrm{R}}{\mathrm{d}}\right) \Delta \mathrm{RD}^{3} \mathrm{j}_{\nu^{\prime}}\right] \mathrm{d} \nu
$$

where $\Delta R$ is the width of the shock region and is assumed to be $\Delta R=R / 10$, after Wang et al. (2003) in the calculation.

To reduce the number of free parameters, we set $\gamma_{m}=100$ in our calculation because the results are quite insensitive to this value. According to our kinematic model in section 4.1, we choose the time that the reverse shock takes place to be the time that the Lorentz factor reduced to $1 / \sqrt{2}$ of its original value, which is called deceleration timescale $t_{\mathrm{dec}}$ in the GRB external shock model, which is supposed to be the strongest point of the external shock (Private communication with X. Y. Wang). Then we fit the data to find out the initial values of $K$ and $B_{\perp}$.

Just like the fitting to the kinematics of the jets, we could not decide the best fitting result only using the flux data since we could always find one group of parameters that fit the flux data approximately well for each parameter $r$. Thus, we combine the kinematic and light curve fitting together to find some more useful hints.

We know that the energy and the number density of the gas in the pre-shock and post-shock regions are connected by the jump conditions $n^{\prime}=\zeta(\Gamma) n$ and $e^{\prime}=\eta(\Gamma) n m_{p} c^{2}$, where $\zeta(\Gamma)$ and $\eta(\Gamma)$ are coefficients related to the jet velocity (Wang et al. 2003 ). Therefore if we assume the shocked electrons and the magnetic field acquire constant fractions $\left(\epsilon_{\mathrm{e}}\right.$ and $\left.\epsilon_{B}\right)$ of the total shock energy, we have

$$
\gamma_{m}=\epsilon_{e} \frac{p-2}{p-1} \frac{m_{p}}{m_{e}}(\Gamma-1), K=(p-1) n^{\prime} \gamma_{m}^{p-1},
$$

and

$$
B_{\perp}=\sqrt{8 \pi \epsilon_{B} e^{\prime}}
$$

for $\mathrm{p}>2$. Since we have assumed that $p$ and $\Gamma_{0}$ (so that $1 / \sqrt{2} \Gamma_{0}$ ) are equal for the two jets, if we further assume that factor $\epsilon_{e}$ of the eastern and the western jets is also the same, we may infer that $K \propto e^{\prime} \propto n$ for the two jets. We therefore search for the combination of parameters that could satisfy the kinematic and light curve fitting, as well as the relationship $K_{\mathrm{e}} / K_{\mathrm{w}} \sim n_{\mathrm{e}} / n_{\mathrm{w}}$.

To search for the best parameters fitting the data, we follow the following procedure. With numerical calculations, for the 19 data points (including 10 kinematic data and 9 light curve data) we build a large 8-dimensional database for the eight parameters to be estimated, with one constraining relationship, $K_{\mathrm{e}} / K_{\mathrm{w}} \sim n_{\mathrm{e}} / n_{\mathrm{w}}$. Intuitively it looks very difficult to determine so 
many parameters from so few data points. However, one approach we take is that we divide the fittings into two stages: we first fit the kinematic data regardless of the lightcurve information and get a series of $(r, n)$ all of which describe the data almost equally well. Then, for each set of $(r, n)$, we fit the lightcurve and calculate the joint $\chi^{2}$ value to choose the group of parameters yielding the least $\chi^{2}$ value. The advantage of this approach is that, since $(r, n)$ is fixed every time in the fitting of the lightcurve, we consider the kinematic fittings to be independent of the lightcurve fittings in calculating $\chi^{2}$. At each search step, the total $\chi^{2}$ is calculated corresponding to the 19 data points.

A set of parameters that yields the minimum total $\chi^{2}$ is taken as our best fitting parameters. The resulting total $\chi^{2}$ is 7.05 and the errors are estimated by searching for the range for one parameter that can change the $\chi^{2}$ value by a given delta while keeping all the other parameters fixed (Press et al. 1992, "Constant Chi-Squared Boundaries as Confidence Limits"). The result corresponding to the lightcurve fitting is shown in Fig.5 and the corresponding kinematic fitting is shown in panel (c) in Fig.4. The best fitting parameters are listed in Table 4. From the parameters, we conclude that the boundary of the cavity lies at $r \sim 12 \operatorname{arcsec}$ to the east and $\sim 17$ arcsec to the west of the central source. The corresponding number density of the ISM outside this boundary is $\sim 0.0034 \mathrm{~cm}^{-3}$ and $\sim 0.12 \mathrm{~cm}^{-3}$, respectively. These values are both lower than the canonical ISM value of $\sim 1 \mathrm{~cm}^{-3}$, although the value in the western region is much higher than in the eastern region. The asymmetry of the density on the both sides probably involves the generation history of the cavity, which should be explored with further studies. The electron energy fraction relationship is satisfied as $K_{\mathrm{e}} / K_{\mathrm{w}} \sim n_{\mathrm{e}} / n_{\mathrm{w}} \sim 0.03$. However, we should mention here that the other relation concerning the magnetic field strength (if $\epsilon_{B}$ are equal on the two sides, then $\left.B_{\mathrm{e}} / B_{\mathrm{w}} \propto \sqrt{e_{\mathrm{e}}^{\prime} / e_{\mathrm{w}}^{\prime}} \propto \sqrt{n_{\mathrm{e}} / n_{\mathrm{w}}}\right)$ could not be satisfied simultaneously by these parameters. Although the cavity radius and the number density are allowed to vary significantly, the best fitted magnetic field strength remains quite stable $(\sim 0.35-0.55 \mathrm{mG})$. One possible interpretation for this is that the equipartition parameter varies as the physical conditions of the jet varies or equipartition assumptions do not hold here for the magnetic fields since this is not a steady system; an alternative explanation may involve the in situ generation (or amplification) of the magnetic field (e.g., originated from external ISM instead of from the jet itself).

In the fitting, the assumed initial Lorentz factor is $\Gamma_{0}=3$, indicating an initial velocity of $v \sim 0.943 c$. The fixed spectral index, $p=2.2$, is consistent with the value obtained by Xue et al. (2008) in their broadband spectral analysis $(p \sim 2.20-2.31)$. The fitted magnetic field strengths is lower than the value given by Xue et al. (2008) $(B \sim 1-32 \mathrm{mG})$. However, they claimed a large uncertainty in their fittings. We look forward to further analysis to explore this more clearly and take our fitting as a reasonable one at this stage.

\section{ANALYSIS OF JETS IN H1743-322}

H 1743-322 is an X-ray transient first discovered in 1977 (Kaluzienski \& Holt 1977). It was

then classified as a black hole candidate in 1984 based on its X-ray spectral characteristics (White 
Table 3. Spectral Properties of the Western Jet

\begin{tabular}{ccccccc}
\hline \hline & & & \multicolumn{3}{c}{ Powerlaw Fitting Results $^{\mathrm{a}}$} \\
\cline { 5 - 7 } Obs Num & $\begin{array}{c}\text { Time after X-ray } \\
\text { burst (days) }\end{array}$ & $\begin{array}{c}\text { Total Counts } \\
(0.3-8 \mathrm{keV})\end{array}$ & $\begin{array}{c}\text { Counts Rate } \\
(\mathrm{cts} / \mathrm{s})\end{array}$ & $\begin{array}{c}\text { Photon } \\
\text { Index }\end{array}$ & $\begin{array}{c}\text { Reduced } \\
\chi^{2}\end{array}$ & $\begin{array}{c}\text { Flux }^{\mathrm{b}} \\
\mathrm{ergs} \mathrm{cm}^{-2} \mathrm{~s}^{-1}\end{array}$ \\
\hline 4 & 1265 & 419 & 0.016 & $1.75 \pm 0.11$ & 0.91 & $(1.9 \pm 0.4) \times 10^{-13}$ \\
5 & 1355 & 248 & 0.014 & $1.71 \pm 0.15$ & 0.91 & $(1.6 \pm 0.3) \times 10^{-13}$ \\
6 & 1462 & 197 & 0.008 & $1.94 \pm 0.17$ & 0.90 & $(8.6 \pm 1.5) \times 10^{-14}$ \\
7 & 1588 & 114 & 0.005 & $1.81 \pm 0.22$ & 0.80 & $(5.5 \pm 1.0) \times 10^{-14}$ \\
8 & 1856 & 137 & 0.003 & $1.97 \pm 0.20$ & 1.76 & $(3.1 \pm 0.6) \times 10^{-14}$ \\
\hline
\end{tabular}

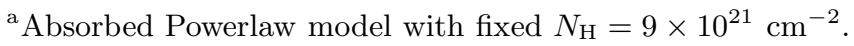

${ }^{\mathrm{b}}$ Absorbed flux in $0.3-8 \mathrm{keV}$ band.

Table 4. Model Fitting Results of the Eastern and Western Jet

\begin{tabular}{|c|c|c|c|c|c|}
\hline & Eastern jet & Western jet & $n_{\mathrm{e}} / n_{\mathrm{w}}$ & $K_{\mathrm{e}} / K_{\mathrm{w}}$ & Comments \\
\hline$r(\operatorname{arcsec})$ & $12 \pm 0.3$ & $17 \pm 0.1$ & & & Cavity radius \\
\hline$n\left(\mathrm{~cm}^{-3}\right)$ & $(3.4 \pm 0.3) \times 10^{-3}$ & $0.12 \pm 0.01$ & & & ISM density outside the cavity \\
\hline$\theta\left({ }^{\mathrm{o}}\right)$ & 68 & 68 & & & Line of sight angle \\
\hline$r_{0}(\operatorname{arcsec})$ & 15.0 & 17.9 & 0.03 & 0.03 & Reverse shock hitting place $^{\mathrm{a}}$ \\
\hline$t_{0}$ (days) & 343 & 842 & & & Reverse shock hitting time ${ }^{a}$ \\
\hline$B_{\perp}\left(t_{0}\right)(\mathrm{mG})$ & $0.51 \pm 0.03$ & $0.38 \pm 0.003$ & & & Initial value at $t_{0}$ \\
\hline$K\left(t_{0}\right)\left(\mathrm{cm}^{-3}\right)$ & $(9.0 \pm 1) \times 10^{-3}$ & $0.32 \pm 0.03$ & & & Initial value at $t_{0}$ \\
\hline \multirow[t]{2}{*}{$\chi^{2}$ for fitting } & kinematics: 1.46 & kinematics: 1.55 & & & total $\chi^{2}=7.05$ reaches the minimum \\
\hline & light curve: 1.68 & light curve: 1.68 & & & \\
\hline
\end{tabular}

${ }^{a}$ The place and time that the Lorentz factor reduced to $1 / \sqrt{2}$ of its original value, according to the kinematic model. 


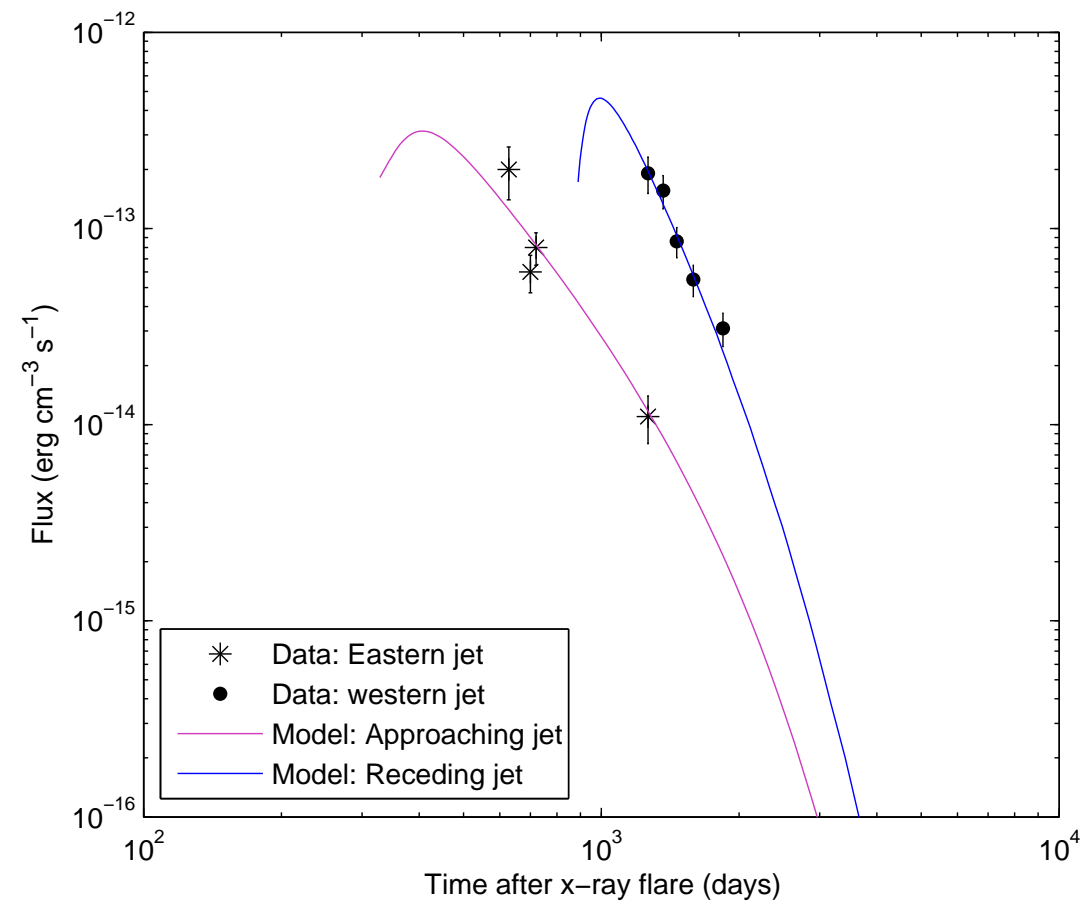

Fig. 5.- Model fittings to the X-ray light curves of the eastern and western jets. A power-law plus Galactic absorption spectral model is used to obtain the energy flux in the $0.3-8 \mathrm{keV}$ band. The two solid lines are the theoretical model fittings for the reverse shock heated ejecta emission. 
\& Marshall 1984). New activity was found by INTEGRAL in 2003 (Revnivtsev et al. 2003), and a bright radio flare was observed by VLA on 2003 April 8 (Rupen et al. 2003). Chandra X-ray and ATCA radio observations from 2003 November to 2004 June revealed the presence of largescale jets on both sides (Rupen et al. 2004; Corbel et al. 2005). The source is then labeled as a "microquasar".

We take the data from the work of Corbel et al. (2005) and fit the proper motion with the external shock model. Following their approach, the ejection date is set to the time of the major

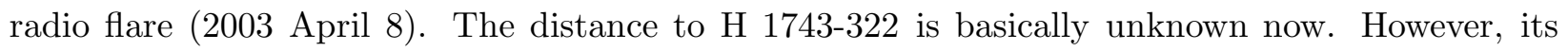
location toward the Galactic bulge could possibly imply a Galactic center location. Assuming a source distance of $8 \mathrm{kpc}$ (distance to the Galactic center), the first radio detection of the jets gives out an intrinsic velocity of the ejection of $\beta=v / c=0.79$ and an angle of $\theta=73^{\circ}$ for the axis of the jets (Corbel et al. 2005).

However, the evolution of the jets shows that the velocity is not constant all the way through. Deceleration is required since the linear extrapolation of the proper motion data yields an ejection date earlier than the chosen zero point time, as shown in panel (a) in Fig. 6. The linear extrapolation of the zero point and the first data point also gives out lines with steeper slope than the real data.

As a result, we also apply the external shock model to this source. The line of sight angle is set to be $\theta=73^{\circ}$ in all fittings. It is worth mentioning that the 73 degree angle is only valid if the jets have both been propagating outwards with the same speed and the same angle to the line of sight and the source is assumed to be located at the Galactic center with a distance of $8 \mathrm{kpc}$. However, since there are no better constraints on the jet orientation, we simply take this value as a reasonable approximation. We first test the model that assumes the source located in a continual gas medium. In this case, the jets would decelerate gradually after ejection. The model fits the data quite well this time (panel (b) in Fig.6, with $\chi^{2}=1.03$ for the approaching jet and $\chi^{2}=0.98$ for the receding jet, with degree of freedom $=2$ for each case), indicating that a cavity is not required in this source. However, we also notice that the best fitting density of the environment ISM is $n \sim 3 \times 10^{-4} \mathrm{~cm}^{-3}$, much lower than the canonical Galactic ISM value. We also apply the cavity model to the fitting, which describes the data equally well (panel (c) in Fig. 6, with $\chi^{2}=0.95$ for the approaching and $\chi^{2}=0.97$ for the receding jet, with degree of freedom $=2$ for each case), with a cavity region with radius of 3 arcsec on both sides and the gas density of $n \sim 3 \times 10^{-3} \mathrm{~cm}^{-3}$. This value is one magnitude higher than the previous attempt, but still quite low. Due to the limited data, we cannot test if the environment outside the central source is symmetric or not. The flux is not fitted for this source because of the limited number of X-ray data points. 

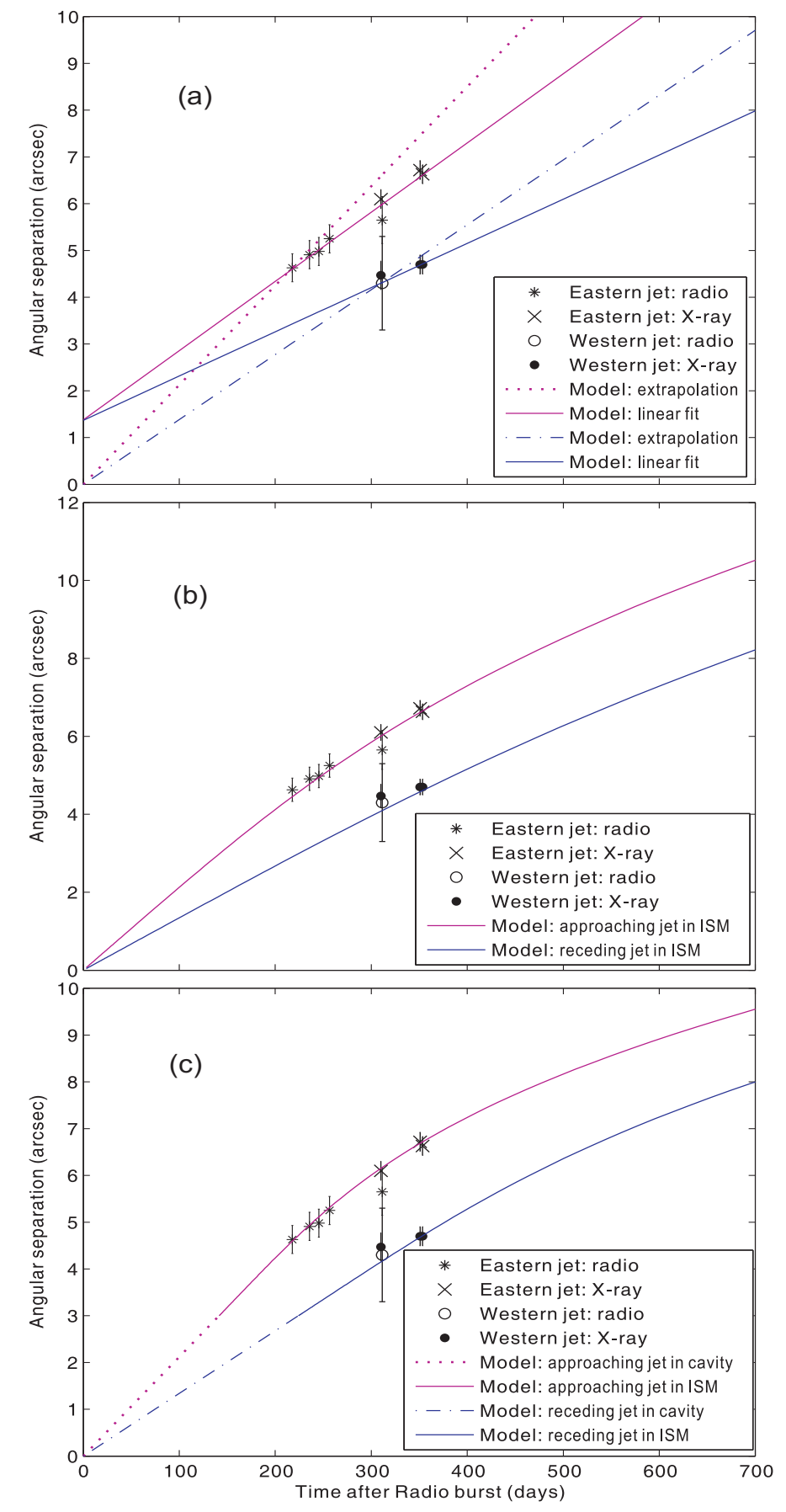

Fig. 6. - Model fittings to the proper motion of the radio and X-ray jets of H 1743-322. Panel (a) shows the constant velocity fitting and Panel (b) and (c) are deceleration model fittings. Panel (a): Dotted lines: extrapolation of the zero point time and the first radio detection. Solid lines: linear fittings to the data set with constant velocity. Panel (b): Deceleration in constant density medium. Results: $\Gamma_{0}=1.65, \theta=73^{\circ}, E_{0}=1 \times 10^{44} \mathrm{erg}, n \sim 3 \times 10^{-4} \mathrm{~cm}^{-3}$. Panel (c): Deceleration in medium outside a cavity region. Results: $\Gamma_{0}=1.65, \theta=73^{\circ}, E_{0}=1 \times 10^{44} \mathrm{erg}, r_{\mathrm{e}}=r_{\mathrm{w}}=3 \operatorname{arcsec}$, $n \sim 3 \times 10^{-3} \mathrm{~cm}^{-3}$. 


\section{GENERAL PICTURE OF MICROQUASAR ENVIRONMENT}

The above analyses of XTE J1550-564 and H 1743-322 led us to the conclusion that in microquasars the interactions between the ejecta and the environmental gas play major roles in the jet evolution. We could further argue that the low density of the environment is a necessary requirement for the jet to develop to a long distance.

Heinz (2002) derived the scale relations for the jets from accreting black holes, using a simple analytic model. He adopted the jet-ISM interaction scenario to estimate the slow-down distance of the ejecta:

$$
d_{\text {slow }} \sim 10^{16} \mathrm{~cm}\left(\mathrm{E}_{44} / \Gamma_{5}^{2} \mathrm{n}_{\mathrm{x}} \theta_{5}^{2}\right)^{1 / 3},
$$

where $E_{\text {kin }} \equiv 10^{44} E_{44}$ erg is the kinetic energy, $\Gamma_{5} \equiv \Gamma / 5$ is the Lorentz factor of the jet, $n_{x}$ is the external gas density in the unit of $\mathrm{cm}^{-3}$ and $\theta=5^{\circ} \theta_{5}$ is the opening angle of the jet. He applied this estimation to microquasars GRS 1915+105 and GRO J1655-40. In both cases, he found that the upper limit on the gas density is roughly $10^{-3} \mathrm{~cm}^{-3}$, given $d_{\text {slow }}$ of $0.05 \mathrm{pc}$, quite consistent with the ISM density found for XTE J1550-564 and H1743-322 in this work. For a larger $d_{\text {slow }}$, a lower $n_{x}$ should be required.

Heinz's approach led to an interesting comparison between the microquasar jets and the radio quasar jets. He claimed that the jets length in GRS 1915+105 ( $l>0.04 \mathrm{pc})$ would correspond to a jet length of $4 \mathrm{Mpc}$ when scaled by $M$ (mass of the central black hole) to AGN conditions (such as M87 or Cyg A) (2002). We could follow this way and infer that the jet in XTE J1550-564 ( $l>0.5$ pc) will correspond to a $50 \mathrm{Mpc}$ long AGN jet, which has never been detected. This again confirms that the environment of microquasars should be comparatively vacuous, in a dynamical sense even (although not in the absolute sense) less dense than the AGN environment (the intergalactic medium of densities between $10^{-5} \mathrm{~cm}^{-3}$ to $10^{-2} \mathrm{~cm}^{-3}$ ), when compared to the thrust of the jets in microquasars and AGNs. When presented in unit of pc with the information of source distance and scaled by the central black hole mass, the proper motions of different microquasar jets can be plotted in one figure (Fig. 7) (GRS 1915+105: 14 $\pm 4 M_{\odot}$ (Greiner et al. 2001), $12.5 \mathrm{kpc}$ (Rodríguez et al. 1995); GX 339-4: $5.8 \pm 0.5 M_{\odot}$ (Hynes et al. 2003), lower limit estimation of distance of $4 \mathrm{kpc}$ (Zdziarski et al. 1998); XTE J1550-564: 10.5 $\pm 1.0 M_{\odot}$ and $5.3 \mathrm{kpc}$ (Orosz et al. 2002); H 1743-322: since no good mass and distance observation up to date, we follow the assumption of $10 M_{\odot}$ (Miller et al. 2006) and galactic center origin of $8 \mathrm{kpc}$ (Corbel et al. 2005)). It is interesting to notice from this figure that although the detailed properties of the binary systems (e.g., the compact objects and the companion stars) differ a lot from source to source, the normalized jet proper motions are quite consistent. This is not surprising since the jet properties are set by the compact object (and its immediate neighborhood) and their evolutions are mostly influenced by the surrounding environment. Although the companion star provides the material which is eventually launched into the jets, and thus affects the mass transfer rate and the mass flux in the jets, it nevertheless does not seem to play significant roles for the structure and evolution of the large scale jets and cavities surrounding the microquasar. Therefore the nature of accretion disk physics dominates over the 
nurture of the companion star, as far as the jets are concerned. Thus it leads us to the attempt to generalize the microquasar jets in one consistent picture.

This figure, together with other observational data concerning the motionless large scale jets (e.g., Cyg X-1, SS433), has led us to the suggestion that all microquasar jets can be classified into roughly three groups: small scale moving jets, large scale moving jets and large scale jet relics. For the first type, the "small jets", only radio emissions are detected. The jets are always relatively close to the central source and dissipate very quickly. Examples of this class include GRS 1915+105 (Rodríguez \& Mirabel 1999; Miller-Jones et al. 2007), GRO J1655-40 (Hjellming \& Rupen 1995), and Cyg X-3 (Martí et al. 2001). The typical spatial scale is $0 \sim 0.05$ pc and the time scale is several tens of days. In this class, no obvious deceleration is observed before the jets become too faint. For the second type, the "large jets", both X-ray and radio detections are obtained, at a place far from the central source several years after the outburst. Examples are XTE J1550-564, H1743-322, and GX 339-4 (Gallo et al. 2004). The typical jet traveling distance for this type is $0.2 \sim 0.5 \mathrm{pc}$ from the central engine. Deceleration is clearly observed in this type of sources. The last type, the "large relics", is a kind of diffuse structures observed in radio, optical and X-ray band, often ring or round nebula shaped that are not moving at all. In this class, some well studied sources, Cygnus X-1 (Gallo et al. 2005), SS433 (Dubner el al. 1998), Circinus X-1 (Stewart et al. 1993, Tudose et al. 2006) and GRS 1915+105 (Kaiser et al. 2004) are included. The typical scale for this kind is $1 \sim 30 \mathrm{pc}$, an order of magnitude larger than the second type. The estimated lifetime often exceeds one million years, indicating that they are related to previous outbursts.

From these properties, it is reasonable to further suggest a consistent picture involving all the sources together. We make a conjecture that extreme low density regions, or namely, large scale cavities, exist in all microquasar systems. The "small jets" observed right after the ejection are just traveling through these cavities. Since there are few or none interactions between the jets and the surrounding gas in this region, the jets travel without obvious deceleration. The emission mechanism is synchrotron radiation by particles accelerated in the initial outburst. The emissions of jets decay very quickly and are not detectable after several tens of days. In some cases (e.g. XTE J1550-564), the cavity has a dense (compared to the cavity) boundary at some radius and the interactions between the jets and the boundary gas heat the particles again and thus make the jets detectable again. Those are the "large jets". The emission mechanism then is synchrotron radiation by the re-heated particles in the external shocks. Then, after these interactions, the jets lost most of their kinematic energy into the ISM gradually, causing the latter to expand to large scale structures, the "large relics", in a comparatively long time (thousands to several millions of years) and create the observed nebula-like jet-inflated bubbles.

Different systems are allowed to fall into more than one of the three postulated groups. For example, Cygnus X-1 has small-scale ejecta (Fender et al., 2006) and also has a large-scale relic (Gallo et al., 2005). Also XTE J1550-564 has small-scale ejecta (Hannikainen et al., 2001) and also large-scale jets (Corbel et al., 2002). As we proposed in the above paragraph, the three observational groups are perhaps just three stages in a complete ejection evolution process: the 
"small jets" observed in the outburst events will travel in dark and manifest themselves as the "large jets" when they hit the boundary of the cavity and eventually develop into the "large relics" gradually by the continuous interactions and energy dissipation. If this conjecture holds, then the three groups may not be actually "three" but just three phenomena in "one" system. The three groups identified here are probably just observational manifestations of one generic mechanism, and different sources can belong to multiple groups.

If this general model of microquasars is true, properties of different jet systems could be dealt with separately in one consistent scheme. One implication is that the electrons from the "large jet" and the "small jets" belong to different groups of electrons, from the ejecta itself and the ISM respectively, which means we could not simply use the properties of electron distributions in the "large jet" blobs to determine the emission properties of the central source in the ejection (Xue et al. 2008).

One important remaining question would be why there are such vacuous regions and how to generate them. There are several possibilities, involving previous outbursts, jets or winds activities. Before we go further into these possibilities, we should first distinguish one class from the others. We have used the term "cavity" all through our discussion, but actually there are two geometric possibilities for a cavity. One is a "cavity", the other is a "tunnel". Instead of a spherical cavity, it is possible that there is only a vacuum conical path outside the central engine. It may be created by previous jet ejection events and got fully developed through a series of ejections. This could explain the collimation of the jets naturally, but it requires almost continuous jet ejections (contrary to the episodic jet ejections discussed here) to prevent the surrounding gas to fall back and fill up the thin path between ejections.

This assumption is of great interest because it provides a possible connection between the large-scale structures and the persistent, low-power, steady jets reported in several important observations (etc. Cyg X-1 (Stirling et al. 2001) and GRS 1915+105 (Dhawan et al. 2000)). Kaiser and Alexander (1997, hereafter KA97) has developed a model for radio galaxies in which continuous and collimated jet may clear its way out and create a hot spot at the end of its path, and the hot electrons escaped from the shocks would form a "cocoon" outside the central compact object. If this is also the case for Galactic accretion systems, as postulated for Cygnus X-1 (Gallo et al., 2005), Circinus X-1 (Tudose et al., 2006), and GRS 1915+105 (Kaiser et al., 2004), then the vacuous regions (tunnel and cocoon) and the "large relics" are perhaps both the results of the continuous jets traveling in dark. The two Galactic center sources 1E1740-294 and GRS 1958-258 may also belong to this case. In this frame of assumptions, the episodic outbursts are not included here: the outbursts will increase the instantaneous mass flux down the jet, however since low-mass $\mathrm{X}$-ray binaries spend the majority of their time in a low-luminosity quiescent state, the outbursts will have little effect on the time-averaged jet luminosity, and thus on the long-term evolution of the relics. However, we may infer that if there are dark continuous jets and dark relics in a system like XTE J1550-564, a strong episodic outburst would just hit and brighten the boundary of the relic and may provide a bridge between the source and the relic. If there are multiple outbursts, 
we will expect repeated "large jets" in the same direction with larger and larger lengths. Future observations would then be able to testify this possibility.

Apart from this case, several other possibilities may take place. One possible way to create the observed cavities is through recent supernovae outbursts that produced the central black holes. However, only HMXBs should be close to their parent supernova remnants (being young); accumulated velocity dispersion (scattering from spiral arms or giant molecular clouds over time) will carry LMXBs far from their natal supernova remnant in a few Galactic orbits, e.g. for GRO J1655-40 (Israelian et al. 1999). Furthermore, some sources such as Cygnus X-1 most likely never had supernovae, because of their low peculiar velocity (Mirabel \& Rodrígues 2003). In the case of recent supernovae $\left(<10^{5}\right.$ years) and low kick-out velocity for the black hole, SNRs surrounding them should have been detected, and for much older supernovae and very low kick-out velocity for the black hole, the microquasars may still resides in the SNRs but regular interstellar medium should have filled those very old SNRs now.

Winds from progenitors of the central compact objects at their last stages may also be an interpretation. Cavities in hydrogen maps have been detected around neutron stars and were supposed to be driven by wind from the progenitors of the stars (Gaensler et al. 2005). However, the lifetime of such bubbles were estimated to be of order of $\sim 1$ Myrs. For a region still as vacuous as we have estimated in our studies, the compact object is expected to be much younger than that. Winds of the companion star in the binary are also a possible candidate. Wind-blown bubbles and shells are observed around evolved massive stars, with typical radii of $\sim 2-10$ pc for Wolf-Rayet stars (Gruendl et al. 2000) and $\sim 0.1-2.3$ pc for luminous blue supergiants (Smith et al. 2007). However, the estimated density at the bubble shells is of order of $\sim 100 \mathrm{~cm}^{-3}$, simply too high for our case, and among all the well established microquasar systems, only the companion star in Cyg X-1 is $\mathrm{O}$ type (O9.7Iab) (Remillard \& McClintock 2006), whereas most of the other systems contain only M, $\mathrm{K}$ or $\mathrm{G}$ type companion stars, which are not powerful enough to generate such large scale bubbles.

A plausible possibility involves the accretion disk winds. In this scenario, it is the disk winds with mild or non-relativistic velocities that have pushed away the surrounding ISM gas and created the low density regions surrounding the central source. The wind should go as shells that travel in a ballistic way while interacting with the ISM cold gas. Indeed, in several microquasars, fast ionized winds have already been observed (Miller et al. 2006; Kotani et al. 2000; Netzer, 2006; Sala et al. 2006; Fuchs et al. 2006). Strong absorption metal lines are detected with obvious blueshift. The speed of the outflow could be calculated from the blueshift of these lines. The ejection place could also be estimated using the measured ionization parameters. We list some of the results in Table 5, including the calculated escape velocity at the inferred ejection radius $\left(r_{\mathrm{g}}=G M_{\mathrm{BH}} / c^{2}\right.$ and $\left.v_{\mathrm{esc}}(r)=\sqrt{2 G M_{\mathrm{BH}} / r}=\sqrt{2 r_{\mathrm{g}} c^{2} / r}\right)$. Also, in another interesting work, Fuchs et al. (2006) showed that there are indeed strong subrelativistic winds coming out of the SS 433 system, very much alike the winds from a WR star or from a thick torus or envelope outside the accretion disk and the central object. The estimated mass lost rate is $4.7-7.3 \times 10^{-5} M_{\odot} \mathrm{yr}^{-1}$ for discontinuous winds and 3 times higher for continuous winds, much higher than the observed jet mass loss of 


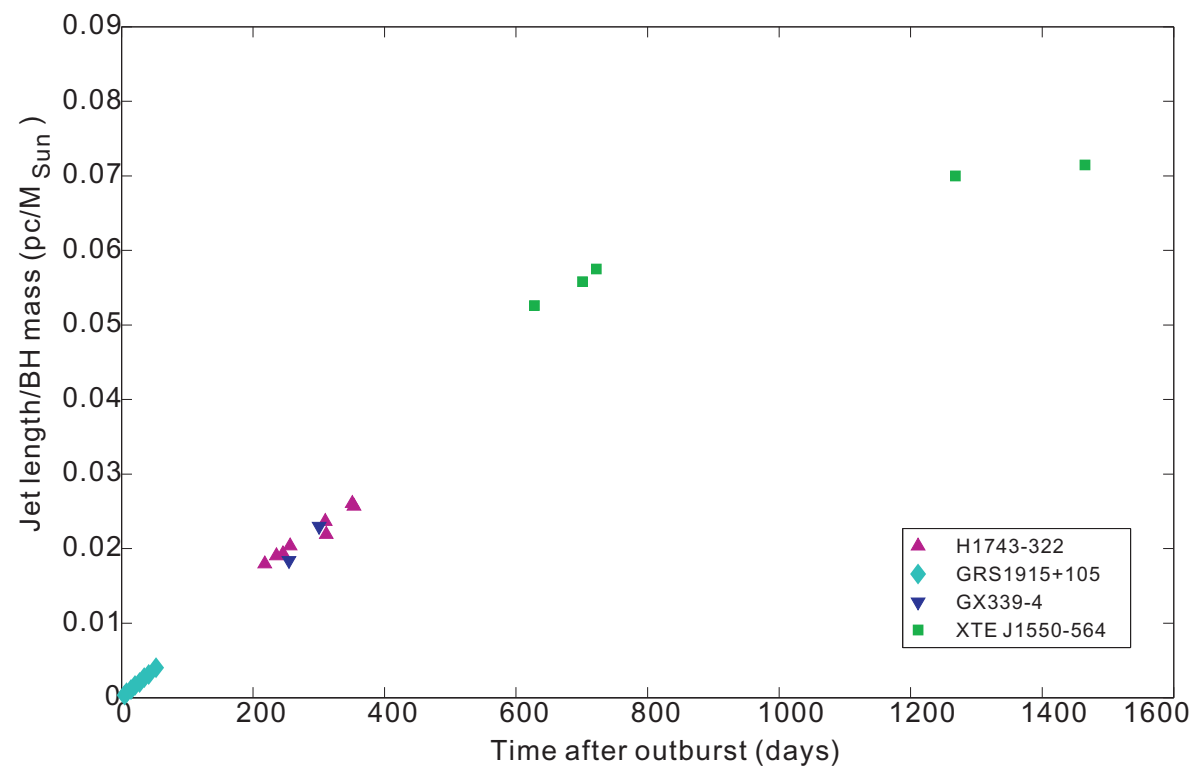

Fig. 7.- Normalized proper motions of microquasars' approaching jets, obtained by dividing the actual jet length by the central compact object mass. For the mass and distance estimations, please refer to the text, see section 6. Jet kinematics data are taken from Rodriguez \& Mirabel (1999), Gallo et al. (2004), Corbel et al. (2005) and this paper respectively.

Table 5. Wind properties of microquasars

\begin{tabular}{|c|c|c|c|c|c|c|c|}
\hline Source Name & Equip. & $\mathrm{M}\left(M_{\odot}\right)$ & $r_{\mathrm{g}}(\mathrm{km})^{1}$ & Velocity $(\mathrm{km} / \mathrm{s})$ & Line width $(\mathrm{km} / \mathrm{s})$ & $R_{\text {eje }} / r_{\mathrm{g}}$ & $V_{\text {esc }}(\mathrm{km} / \mathrm{s})^{2}$ \\
\hline \multirow[t]{4}{*}{ GRO J1655-40 } & Chandra $^{3}$ & 7 & 10.5 & $300-1000$ & $300-500$ & $10^{4.7}$ & 1900 \\
\hline & & & & & & $10^{5.7}$ & 600 \\
\hline & XMM-Newton ${ }^{4}$ & & & $2600-4500$ & & $5 \times 10^{3}$ & 6000 \\
\hline & & & & & & $2 \times 10^{4}$ & 3000 \\
\hline H $1743-322$ & Chandra \& $\mathrm{RXTE}^{5}$ & 10 & 15 & $700 \pm 200$ & $1800 \pm 400$ & $10^{4}$ & 4240 \\
\hline \multirow{2}{*}{ GRS $1915+105$} & $\mathrm{ASCA}^{6}$ & $14 \pm 4$ & 21 & 1000 & & $10^{5}$ & 1340 \\
\hline & Chandra $^{7}$ & & & $1100_{-300}^{+360}$ & $980_{-420}^{+450}$ & $3 \times 10^{5}$ & 770 \\
\hline
\end{tabular}

Note. - 1. $r_{\mathrm{g}}=G M_{\mathrm{BH}} / c^{2} ; 2 \cdot V_{\mathrm{esc}}(r)=\sqrt{2 G M_{\mathrm{BH}} / r} ; \mathbf{3}$. Netzer, 2006; 4. Sala et al. 2006; 5. Miller et al. 2006; 6. Kotani et al. 2000; 7. Neilsen \& Lee 2009; 
$\sim 10^{-7} M_{\odot} \mathrm{yr}^{-1}$ in this system, and a double-cone structure may be formed by these winds. These inferences are also supported by Blundell et al. (2001), where the equatorial radio emission seen in SS433 was taken as evidence for a disc wind. These observations support the idea of co-existence of jets and winds in the microquasars systems. We also notice that the wind velocity in H1743-322 is lower than those in GRS $1915+105$ and GRO J1655-40, suggesting larger cavities are formed for the latter two sources than for the former one. It is consistent with the fact that no decelerating jets have been observed in these two sources up to now.

However, whether the winds are powerful enough to create such large cavities in all cases for all sources is unclear at this stage. The terminal places of the wind shells (which determine the size of the cavity) are determined by the wind energy and geometry, specifically, the wind velocity at the ejection place and the opening angle, and the interaction conditions between winds and the gas. These parameters are still very uncertain nowadays because of the lack of enough studies and the densities of the winds are only estimated at the foot of the ejection place. We would roughly estimate that, in order for the wind to move to large radii far from the black hole, the wind velocity should be quite close to the escape velocity at the ejection radius. From table 5, we can see that most of the observed wind velocities are comparable to, although not as large as, the local escape velocity, with the exception of the recently discovered disk wind of about its escape velocity in GRS $1915+105$ in its soft state, with mass loss rate sufficient to suppress jet ejection (Neilsen \& Lee 2009). Therefore in the last case the accretion disk wind alone (because no jet is expected in the soft state) may play a significant role for forming a large scale cavity. However for other cases the winds alone may not be enough to power the cavities, due to insufficient velocities.

Therefore some kinds of acceleration or velocity-maintaining mechanisms may be required, in order for the winds to expand far enough $(\sim 0.1 \mathrm{pc}$ to $10 \mathrm{pc})$. Magnetic fields may play an important role here. It has been known that rotating wind-up magnetic fields can accelerate and launch jets (see Spruit 2008 and references therein); therefore continuous high velocity winds can set up the favorable condition for producing continuous outflows or jets with velocities around or even exceeding the escaping velocities of these systems. Once continuous high velocity outflows or jets are produced, they should be able to inflate their surrounding interstellar medium to form bubbles, as we have discussed earlier within the framework of the KA97 model.

Finally, we comment that it seems counter intuitive that the boundary of the cavity lies further in the western side, yet the ISM density is also higher in the west. It would be a problem if the cavity is produced by the observed large scale two-sided jets with equal power. Therefore this asymmetry may be used to argue against such a model. However, the asymmetric cavity may be naturally produced if the system has a non-negligible space velocity with respect to the local ISM along the eastern direction. In this case additional energy is injected into the ISM on the head-on side along the direction of motion of the system, in analogy to process producing the head-on bowshock of young spin-down powered pulsars moving in ISM (e.g., Caraveo et al 2003). Therefore the cavity on the approaching side should be emptier (with smaller density) than the opposite wind or jet, unless the system moves perpendicular to the wind or jet. The system itself is also catching up 
with the cavity on the approaching side, placing itself closer to the boundary of the cavity on the

approaching side. For example, a space velocity of $100 \mathrm{~km} / \mathrm{s}$ with respect to the local ISM would displace the source by $0.1 \mathrm{pc}$ in less than 1000 years. In the absence of data for this system on its proper motion and the power of wind or continuous (dark) jets, we will not speculate further on what combination of parameters may eventually produce the observed asymmetry of the cavity. This should be tested in the future. Heinz et al. (2008) have proposed that the microquasar's space velocity should be important and leave trails behind their moving path in the Galaxy. They estimated the volume of radio plasma released by these microquasars and claimed that they should be observable at low frequencies. Future observational confirmation of their prediction for XTE J1550-564 would provide evidence for our conjecture.

\section{CONCLUSIONS AND PERSPECTIVES}

Two large-scale X-ray jets have been observed in XTE J1550-564. We have analyzed the Chandra X-ray data for these two jets and fitted their kinematics and light curves with the external shock model in the GRB afterglow model. In this model, the interactions between the jet material and the surrounding ISM slow the jets down and accelerate the jet particles to radiate the observed radio and X-ray synchrotron radiations. Under such assumptions, the number density of the ISM in the surrounding regions of the central black hole is a key parameter. We found in the fitting that this number density has to be extremely low, or the strong interactions would block the jet's expansion. However on the other hand, the number density at $\sim 20$ arcsec has to be comparatively high (but still lower than the typical ISM) in order to allow the jet to be decelerated quickly, as the observational data indicated. Thus, a model consisting of two different regions, a cavity and its gas boundaries, is tested and shown to work well. The cavity is found to be large scale $(\sim 0.4$ pc in size) and asymmetric $\left(n_{\mathrm{e}} / n_{\mathrm{w}} \sim 0.03\right)$. The reason of the asymmetry of the environment on the two sides is not clear from current observational data. The deceleration described in the model is quite obvious (from $\Gamma_{0}=3$ to $\Gamma_{t} \simeq 1$ at $t \sim 1500$ days), thus it is the first time that we observe the whole process of the deceleration of a pair of relativistic jets.

The similar analysis of H1743-322 also supports this jet-ISM interaction scenario. Large scale decelerating jets are also observed in this source and the interaction model describes the data consistently. Whether a cavity exists is not clear in this case; nevertheless the density is also found to be very low, compared to the canonical Galactic value.

With these studies, a generic model of the environment of microquasars is proposed. We suggest that microquasars are located in large scale and very low density cavities. The cavity provides the space for the jets to go through freely and the interactions between the jets and the material outside the cavity provide the deceleration mechanism of the jets. The cavity is likely created by continuous high velocity outflows or jets, which are seeded by accretion disk winds that have been observed in several microquasar systems producing strong absorption lines. 
Microquasars are powerful probes of both the central engine and their surrounding environment. More studies of the jets behaviors may give us information on the ISM gas properties, as well as the ejecta components. The link between jets and wind is a key issue in current studies of accreting systems. If the general scenario we have proposed here is further confirmed, we then need to understand the mechanism producing these outflows (winds and jets) and the connections between them. Detailed analysis of the geometry and timing properties of the ejecta in the future would be of great significance; it will not only provide insights of the jet formation process, but also offer another approach into black hole physics and accretion flow dynamics.

\section{Acknowledgments}

We are grateful to X.Y. Wang for many helps in this work. We also thank Z.G. Dai and R. Soria for useful discussions. We appreciate very much the insightful comments and helpful suggestions by the anonymous referee. SNZ acknowledges partial funding support by the Yangtze

Endowment from the Ministry of Education at Tsinghua University, Directional Research Project of the Chinese Academy of Sciences under project No. KJCX2-YW-T03 and by the National Natural Science Foundation of China under grant Nos. 10821061, 10733010, 10725313, and by 973 Program of China under grant 2009CB824800.

\section{REFERENCES}

Blandford, R. D., \& McKee, C. F. 1976, Phys. Fluids, 19, 1130

Blundell, K. M., Mioduszewski, A. J., Muxlow, T. W. B., et al. 2001, ApJ, 562:L79

Caraveo P. A. et al. 2003, Science, 301, 1345

Corbel S., Kaaret P., Jain R. K., et al. 2001, ApJ, 554, 43

Corbel, S., Fender, P. R., et al. 2002, Science, 298, 196

Corbel, S., Kaaret, P., et al. 2005, ApJ, 632, 504

Dhawan, V., Mirabel, I. F., Rodrígues, L. F. 2000, ApJ, 543, 373

Dickey, J. M., \& Lockman, F. J. 1990, ARA\&A, 28, 215

Dubner, G. M., Holdaway, M., Goss, W. M., \& Mirabel, I. F. 1998, AJ, 116, 1842

Freeman, P. E., Kashyap, V., Rosner, R., \& Lamb, D. Q. 2002, ApJS, 138, 185

Fuchs, Y., Miramond, L. K., Ábrahám, P. 2006, A\&A, 445, 1041

Gaensler, B. M. et al. 2005 ApJ, 620, L95 
Gallo, E., Corbel, S., Fender, R. P., et al. 2004, MNRAS, 347, L52

Gallo, E., Fender, R. P., Kaiser, C., et al. 2005, Nature, 436, 819

Greiner, J., Cuby, J. G., \& McCaughrean, J. 2001, Nature, 414, 522

Gruendl, R. A., Chu, Y. H., Dunne, B. C., \& Points, S. D. 2000, AJ, 120, 2670

Hannikainen, D., Campbell-Wilson, D., Hunstead, R., et al. 2001, ApSS Supp., 276, 45

Heinz, S., 2002, AA, 388, L40

Heinz, S., Grimm, H. J., Sunyaev, \& R. A., Fender, R. P., 2008, ApJ, 686, 1145

Hjellming, R.M., \& Rupen, M. P. 1995, Nature, 375, 464

Huang, Y. F., Dai, Z.G., \& Lu, T. 1999, MNRAS, 309, 513

Hynes, R. I., Steeghs, D., Casares, J., Charles, P. A., O’Brien K., 2003, ApJ, L583

Israelian, G., Rebolo, R., Basri, G., et al. 1999, Nature, 401, 142

Jain, R. K., Bailyn, C. D., Orosz, J. A., et al. 2001, ApJ, 554, L181

Kaluzienski, L. J., \& Holt, S. S. 1977, IAU Circ. 3099

Kaaret, P., Corbel, S., \& Tomsick, J.A. 2003, ApJ, 582, 945

Kotani, T., Ebisawa, K., Dotani, T., et al. 2000, ApJ, 539, 413

Kubota, A., \& Makishima, K. 2004, ApJ, 601, 428

Martí, J., Paredes, J., M., \& Peracaula, M., 2001, A\&A, 375, 476

Massi, M., \& Kaufman Bernadó, 2008, A\&A, 477, 1

Miller, J. M, Raymond J., Homan, J., et al. 2006, ApJ, 646, 394

Miller-Jones, J. C. A., Rupen, M. P., Fender, R. P., et al. 2007, MNRAS, 375,1087

Mirabel, I. F., \& Rodríguez, L. F. 1999, ARA\&A, 37, 409

Mirabel, I. F., \& Rodrigues, I., 2003, Science, 300, 1119

Neilsen, J., \& Lee, J. C., 2009, Nature, 458, 481

Netzer, H. 2006, ApJ, 652, L117

Orosz, J. A., Groot, P. J., van der Klis, M., et al. 2002, ApJ, 568, 845

Press, W. H., Flannery, B. P., et al. 1992, Numerical Recipes in C: The Art of Scientific Computing 
Rybicki, G. B., \& Lightman, A. P. 1979, Radiative Process in Astrophysics (New York: Wiley)

Rees, M. J., and Mészáros, P. 1992, MNRAS, 258, 41

Remillard R. A., McClintock, J. E. 2006, ARA\&A, 44, 49

Revnivtsev, M., Chernyakova, M., Capitani, F. 2003, ATel, 132

Rodríguez, L. F., Mirabel, I. F., \& Martí, J. 1992, ApJ, 401, L15

Rodríguez, L. F., Gerard, E., Mirabel, I. F. et al. 1995, ApJS, 101, 173

Rodríguez, L. F., \& Mirabel, I. F. 1999, ApJ, 511, 398

Rupen, M. P., Mioduszewski, A. J., \& Dhawan, V. 2003, ATel, 142, 1

- 2004, AAS, 204, 516

Sala, G., Greiner, J., Vink, J., et al. 2007, A\&A, 461, 1049

Smith, D. A., 1998, Int. Astron. Union Circ. No. 7008

Smith, N., Bally, J., \& Walawender, J. 2007, AJ, 134, 846

Stewart, R. T., Caswell, J. L., Haynes, R. F., \& Nelson, G. J. 1993, MNRAS, 261, 593

Stirling, A. M., Spencer, R. E., de la Force, C. J., et al. 2001, MNRAS, 327, 1273

Sturner S. J., \& Shrader, C. R. 2005, ApJ, 625,923

Tingay, S. J., Jauncey, D. L., Prestonet, R. A., et al. 1995, Nature, 374, 141

Tomsick, J. A., Corbel, S., \& Fender, R. 2003, ApJ, 582, 933

Tudose, V., Fender, R. P., Kaiser, C. R., Tzioumis A. K. et al. 2006, MNRAS, 372, 417

van der Laan, H. 1966, Nature, 211, 1131

Wang, X. Y., Dai, Z. G., \& Lu, T. 2003, ApJ, 592, 347

White, N. E., \& Marshall, F. E. 1984, ApJ, 281, 354

Xue, Y. Q., Wu, X. B., \& Cui, W. 2008, MNRAS, 384, 440

Yuan, F., Zdziarski, A. A., Xue, Y., \& Wu X.-B. 2007, ApJ, 659, 541

Zdziarski, A. A., Poutanen, J., Mikolajewska J., et al. 1998, MNRAS, 301, 435 